\title{
Fast method for skeletal tissue gene expression analysis
}

\author{
LUCA DALLE CARBONARE ${ }^{1}$, MARIA TERESA VILEI ${ }^{2}$, CHIARA STRANIERI $^{1}$, \\ GIULIO INNAMORATI ${ }^{1}$, ANTONIO ROSATO ${ }^{3,4}$, ELISA BOLDRIN ${ }^{2}$, \\ STEFANIA SELLA $^{2}$, SANDRO GIANNINI $^{2}$ and MARIA TERESA VALENTI ${ }^{1}$ \\ ${ }^{1}$ Department of Medicine, Section of Internal Medicine D, University of Verona, I-37134 Verona; \\ ${ }^{2}$ Clinica Medica 1, Department of Medicine; ${ }^{3}$ Department of Surgery Oncology and Gastroenterology, \\ University of Padova; ${ }^{4}$ Veneto Institute of Oncology-IRCCS, I-35128 Padova, Italy
}

Received February 24, 2016; Accepted May 11, 2016

DOI: 10.3892/br.2016.699

\begin{abstract}
Several chronic diseases have been associated with bone alteration in the last few years. Despite the wealth of information provided by the analysis of the transcriptome in affected tissues, only a limited number of studies evaluated gene expression in bone tissue due to the difficulty to obtain high quality RNA. Therefore, skeletal pathologies have been often associated to a defective maturation process that occurs during recruitment of progenitor stem cells. In order to explore the possibility of analysing the gene expression during osteogenic differentiation in skeletal tissue, a single-step method to extract well-preserved RNA from bone specimens was performed. A comparison between this technique and a traditional method was made by analysing the quality and yield of RNA obtained. In addition, RNAs were assayed by reverse transcription-quantitative polymerase chain reaction to analyse the expression levels of the bone genes associated with the differentiation process in a mouse model. The present data showed that good quality RNA can be obtained from bone tissue by a simple single-step method allowing the expression analysis of the genes encoded by skeletal tissue. In conclusion, the present study allows the possibility to easily obtain good quality RNA from bone tissue that is suitable for gene expression studies of bone diseases.
\end{abstract}

\section{Introduction}

Skeletal disorders are degenerative diseases causing progressive disability that are becoming more prevalent in society. Accurate analysis is key for an optimum achievable diagnosis; however, gene expression studies are currently limited as mineralized tissue prevents the study at the molecular level of

Correspondence to: Dr Maria Teresa Valenti, Department of Medicine, Section of Internal Medicine D, University of Verona, 10 Piazzale Scuro, I-37134 Verona, Italy

E-mail: mariateresa.valenti @univr.it

Abbreviations: FSSM, fast single-step method; TM, traditional method

Key words: RNA, gene expression, bone tissue embedded bone cells. Remodelling is extremely important for skeletal integrity. Bone cells, such as osteoblasts, osteocytes and osteoclasts, act to confer a dynamic equilibrium between bone formation and bone resorption (1). Molecular changes occurring in important processes, such as differentiation, or associated with pharmacological responses, are often studied in vitro using bone cells derived from calvariae or femour.

However, these approaches imply digestion and artifactual conditions, such as incubation in media supplemented with sera and growth factors. As a result, in spite of versatility, even well-conducted studies may deliver imprecise information.

Until now, histomorphometric studies performed to evaluate bone microarchitecture describe the quality and integrity of bone tissue $(2,3)$. By contrast, this method does not allow a correct and timely molecular analysis of skeletal changes during the bone remodeling under endogenous and exogenous stimuli.

The possibility to study molecular changes directly in bone tissue appears intriguing and useful. However, in order to perform studies of gene expression associated with bone tissue, it is important to promptly isolate RNA preserving the quality and integrity.

Current RNA isolation methods from bone tissue are based on multiple steps approaches conducted at low temperatures using liquid nitrogen (4) or beads maintained at temperatures near freezing (5). The quality of RNA isolated by these means is good, but the process is time consuming and the extraction steps have to be performed at an extremely low temperature. The latter aspect, often limits the possibility to isolate RNA in sterile conditions and to prevent RNA contamination.

In order to isolate RNA in a simple and fast manner in a sterile cabinet, a new method that prevents RNA degradation and contamination with a great feasibility for numerous laboratories was developed in the present study.

In addition, the RNA obtained by this fast single-step method (FSSM) was assayed by analysing the expression of osteoblastic (Runx2, Alp and Sparc) and osteoclastic (Tnfrsf11 and $C t s k$ ) genes, and the results were compared with the data obtained using a traditional method (TM) for RNA isolation.

\section{Materials and methods}

Animals. In total, 10 3-month-old female C57BL/6 mice with a body weight of 25-30 g were obtained from Charles River 
Table I. RNA yield and quality for RNA obtained by FSSM and TM.

\begin{tabular}{lcccrr}
\hline Method & Yield,$\mu \mathrm{g}$ & $\mathrm{A}_{260} / 280$ & $\mathrm{~A}_{260} / 230$ & rRNA ratio (28S/18S) \\
\hline TM & $18.22 \pm 2.09$ & $1.98 \pm 0.13$ & $1.95 \pm 0.18$ & $1.84 \pm 0.21$ & $9.23 \pm 0.81$ \\
FSSM & $18.55 \pm 1.48$ & $2.01 \pm 0.01$ & $1.97 \pm 0.14$ & $1.96 \pm 0.19$ & $9.5 \pm 0.22$
\end{tabular}

Yield, rRNA ratio and RIN value were obtained using the RNA 6000 Nano LabChip kit (Agilent 2100 bioanalyzer; Agilent Technologies Inc., Santa Clara, CA, USA) whereas the $\mathrm{A}_{260} / 280$ and $\mathrm{A}_{260} / 230$ by spectrophometer GeneQuant 1300 (GE Healthcare Europe, GmbH, Freiburg, Germany). The values are reported as mean and standard deviation obtained in 10 independent tibias of mice for each method. FSSM, fast simple-step method; TM, traditional method; RIN, RNA integrity number.

Laboratories Italia (Calco, Italy). All the mice were housed under similar conditions. The mice were fed a standard rodent diet containing $0.97 \%$ calcium, $0.85 \%$ phosphorus, $1,045 \mathrm{IU} / \mathrm{kg}$ vitamin D3, 22.5\% protein, $5.5 \%$ fat and $52 \%$ carbohydrate, and were provided access to tap water ad libitum.

The animal procedures were approved by the local government authorities (University of Padova, Italy) and were conducted in accordance with the accepted standards of humane animal care, in accordance with the EU Directive 2010/63/EU.

Tibia bones were harvested and the embedded tissue removed. For each mouse, two tibias were harvested and one tibia for each method was used.

RNA extraction by the FSSM. Soft tissue was removed, the tibia was cut into slices $<0.2 \mathrm{~cm}$ and submerged in RNAlater (Qiagen, Milano, Italy) solution accordingly to manufacturer's protocol, and the samples were incubated overnight at $4^{\circ} \mathrm{C}$. On the following day, RNAlater was removed and the bone was stored at $-80^{\circ} \mathrm{C}$ until RNA isolation.

For isolation, the RNeasy Protect Mini kit (Qiagen) was used with certain modifications and isolation was performed at room temperature.

The slices were thawed at room temperature and simultaneously disrupted and homogenized with a T10 basic ULTRA-TURRAX at $8,000 \mathrm{x}$ g in the presence of $600 \mu \mathrm{l}$ of RLT buffer containing $\beta$-mercaptoethanol in the same microtube used for storage. Subsequently, the lysate was placed into a QIAshredder spin column on a 2-ml collection tube and centrifuged for $2 \mathrm{~min}$ at full speed. The following steps were performed according to the manufacturer's protocol with a DNAse treatment.

RNA extraction by a TM. Total RNA was extracted from each pellet using the RNeasy Mini kit with DNAse treatment. The tibia bones were harvested from mice and any attached tissue was removed prior to the addition of RNAlater solution and stored at $4^{\circ} \mathrm{C}$. After $24 \mathrm{~h}$ the RNAlater was eliminated and the samples were stored at $-80^{\circ} \mathrm{C}$ until RNA extraction. Frozen samples were crushed using a mortar and pestle in liquid nitrogen as previously described (4) and homogenized in RLT Lysis buffer. The RNA isolation was subsequently performed according to the manufacturer's protocol.

RNA evaluation and reverse transcription. The yield and quality of RNA were analysed using the RNA 6000 Nano LabChip kit (Agilent 2100 bioanalyzer; Agilent Technologies Inc., Santa Clara, CA, USA) or the spectrophorometer

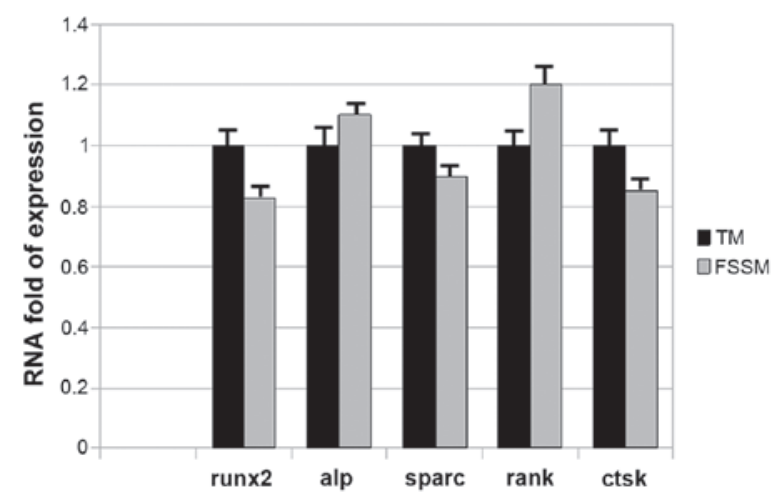

Figure 1. Relative expression levels of the osteoblastic (Runx2, Sparc and Alp) and osteoclastic (Tnfrsfl1 and Ctsk) genes obtained by the FSSM and TM methods. FSSM, fast single-step method; TM, traditional method.

GeneQuant 1300 (GE Healthcare Europe, GmbH, Freiburg, Germany). First-strand cDNA was generated using the High-Capacity cDNA Archive kit, with random hexamers, (Applied Biosystems PE; Thermo Fisher Scientific, Inc., Waltham, MA, USA) according to the manufacturer's protocol. RT product was aliquoted in equal volumes and stored at $-80^{\circ} \mathrm{C}$.

Reverse transcription-quantitative polymerase chain reaction (RT-qPCR). RT-qPCR was performed in a total volume of $50 \mu \mathrm{l}$ containing $1 \mathrm{X}$ TaqMan Universal PCR Master mix, no AmpErase UNG and $5 \mu 1$ of cDNA; gene-specific primers and probe sets for each gene (Runx2, Mm00501584-m1; Alp, Mm01187117-m1; Sparc, Mm00486332-m1; Tnfrsf11a, Mm00437132_m1; Ctsk, Mm00484039_m1) were obtained from Assay-on-Demand Gene Expression Products (Applied Biosystems; Thermo Fisher Scientific, Inc.). In order to normalise the results, two reference genes that belong to different categories: $\beta$-actin (structural gene;) and GAPDH (metabolism-related gene) were used [mouse ACTB (Actin, $\beta$ ) endogenous control, cat. no. 4352341E; and mouse GAPD (GAPDH) endogenous control, cat. no. 4352339E, Applied Biosystems; Thermo Fisher Scientific, Inc., respectively].

Amplifications included $10 \mathrm{~min}$ at $95^{\circ} \mathrm{C}$ (AmpliTaq Gold activation), followed by 40 cycles at $95^{\circ} \mathrm{C}$ for $15 \mathrm{sec}$ and at $60^{\circ} \mathrm{C}$ for $1 \mathrm{~min}$. Thermocycling and signal detection were performed with the ABI Prism 7300 Sequence Detector (Applied Biosystems; Thermo Fisher Scientific, Inc.). Signals were detected according to the manufacturer's protocol. $\mathrm{C}_{\mathrm{q}}$ values were analysed using TaqMan SDS analysis software and triplicate $\mathrm{C}_{\mathrm{q}}$ values were averaged. 
Relative gene expression levels were calculated for each sample following normalization and by the $\Delta \Delta \mathrm{C}_{\mathrm{q}}$ method for comparing relative fold expression differences. The data are reported as mRNA fold expression.

Statistical analysis. Analysis of variance followed by Bonferroni as post-hoc analysis were used and the results are expressed as mean \pm standard error of the mean. $\mathrm{P}<0.05$ was considered to indicate a statistically significant difference. Analyses were applied to experiments carried out at least three times. Statistical analyses were performed using SPSS for windows, version 16.0 (SPSS, Inc., Chicago, IL, USA).

\section{Results and Discussion}

Molecular approaches could provide highly relevant information regarding the basis of degenerative diseases with important consequences on the prevention and treatment. The possibility to obtain specific and sensible tools for diagnosis and follow up of degenerative diseases represents a relevant endpoint to improve and facilitate the managements of patients affected by these diseases. Several degenerative diseases involve the skeletal tissue, a deeper analysis of the bone quality would open the possibility to personalize and improve therapies. However, calcified deposits prevent a direct nucleic acids extraction, which complicates the analysis.

Recently, it has been shown that zoledronate, a potent amino-bisphosphonate, is able to affect peri-implant bone formation (6) but the molecular mechanisms involved in this process are lacking.

A number of studies that aimed to understand the molecular mechanisms of bone remodeling were performed in in vitro models where it is easier to obtain RNA compared to in bone tissue $(7,8)$. However, culture conditions, including medium and sera, have several variations.

The analysis of bone safety with pharmacological treatment is generally evaluated for histomorphometric studies (9). This approach, even if well performed, is not suitable for evaluating molecular changes occurring in osteoblastic and osteoclastic cells during bone remodeling. More in general, the methods used for RNA extraction from bone tissue are time consuming or require certain precautions to avoid molecular degradation (such as performing RNA extraction at low temperatures). However, usually the extraction steps are performed in a sterile cabinet where the presence of ice could cross contaminate the samples.

On the basis of this finding, a simple and standardised method to extract RNA for bone tissue could represent a useful tool for molecular studies in this field.

To compare RNAs prepared either following the method developed in our laboratory (FSSM) or a traditional one (TM), 10 mice were sacrificed. For each mouse 1 tibia was used for FSSM and the other for TM. Yield (reported in $\mu \mathrm{g}$ ) and quality were compared by considering the ratio of absorbances $\left(\mathrm{A}_{260} \mathrm{I}_{280}\right.$ and $\left.\mathrm{A}_{260} \mathrm{I}_{230}\right)$, the rRNA ratio $(28 \mathrm{~S} / 18 \mathrm{~S})$ and the RNA integrity number (RIN) (Table I). The results demonstrated that the two methods produced a good yield of RNA and the RINs, and therefore quality, were high. As compared to previous methods, FSSM does not use a multistep approach (4) and avoids treatment with beads or temperatures below freezing.
Secondly, whether RNA obtained by FSSM is suitable for gene expression studies was examined and RT-qPCR assays were performed. The gene expression analyses were performed using RNA obtained by the two aforementioned different methods. RNA $(1 \mu \mathrm{g})$ was transcribed and $20 \mathrm{ng}$ of cDNA was amplified. The relative expression of osteoblastic (Runx2, Sparc and Alp) and osteoclastic (Tnfrsfll and Ctsk) genes, calculated in 10 preparations for each assay, is reported in Fig. 1. In particular, as a calibrator the mean $\mathrm{C}_{\mathrm{q}}$ for each gene obtained from the cDNA of TM was used and normalised for the mean of the corresponding $\mathrm{C}_{\mathrm{q}}$ of the reference genes, and the fold-change in expression was calculated using $\Delta \Delta \mathrm{C}_{\mathrm{q}}$ of the sample obtained by FSSM. The Runx2, Sparc and Alp genes associated with osteoblastic lineage, and the Tnfrsfll and Ctsk genes associated with osteoclastic lineage were chosen, as they are important targets to skeletal tissue. The fold-change of expression that was obtained by amplifying cDNA either for osteogenic or osteoclastic genes was comparable between the TM and FSSM.

In conclusion, molecular studies in bone tissue are limited due to the difficulty in purifying a high quality of RNA. To bypass this, a single-step method was performed and this technique was compared with a TM. The FSSM carried out in the present study showed a good result demonstrating that it is possible to purify bone RNA in a simply way in order to perform gene expression studies in skeletal diseases.

The FSSM performed in the present study is a convenient and rapid protocol to obtain a high quality of bone RNA. This method could simplify the molecular characterization of bone bioptic samples allowing gene expression studies of skeletal tissue.

\section{Acknowledgements}

The authors would like to thank Dr Samuele Cheri for help with the reagent preparations.

\section{References}

1. Lemaire V, Tobin FL, Greller LD, Cho CR and Suva LJ: Modeling the interactions between osteoblast and osteoclast activities in bone remodeling. J Theor Biol 229: 293-309, 2004.

2. Dalle Carbonare L, Ballanti P, Bertoldo F, Valenti MT, Giovanazzi B, Giannini S, Realdi G and Lo Cascio V: Trabecular bone microarchitecture in mild primary hyperparathyroidism. J Endocrinol Invest 31: 525-530, 2008.

3. Dalle Carbonare L, Valenti MT, Bertoldo F, Zanatta M, Zenari S, Realdi G, Lo Cascio V and Giannini S: Bone microarchitecture evaluated by histomorphometry. Micron 36: 609-616, 2005.

4. Mantila Roosa SM, Liu Y and Turner CH: Gene expression patterns in bone following mechanical loading. J Bone Miner Res 26: 100-112, 2011.

5. Carter LE, Kilroy G, Gimble JM and Floyd ZE: An improved method for isolation of RNA from bone. BMC Biotechnol 12: 5, 2012.

6. Kettenberger U, Ston J, Thein E, Procter P and Pioletti DP: Does locally delivered Zoledronate influence peri-implant bone formation? - Spatio-temporal monitoring of bone remodeling in vivo. Biomaterials 35: 9995-10006, 2014.

7. Khedgikar V, Kushwaha P, Gautam J, et al: Withaferin A: A proteasomal inhibitor promotes healing after injury and exerts anabolic effect on osteoporotic bone. Cell Death Dis 4: e778, 2013.

8. Wu M, Hesse E, Morvan F, et al: Zfp521 antagonizes Runx2, delays osteoblast differentiation in vitro, and promotes bone formation in vivo. Bone 44: 528-536, 2009.

9. Recker RR, Ste-Marie LG, Chavassieux P, McClung MR and Lundy MW: Bone safety with risedronate: Histomorphometric studies at different dose levels and exposure. Osteoporos Int 26: 327-337, 2015. 